\title{
Deployment-related pulmonary symptoms and cardiopulmonary exercise testing in military personnel
}

\author{
Paul D. Hiles ${ }^{1 *}$, William H. Porr ${ }^{2}$, William N. Hannah ${ }^{3}$ and MichaelJ. Morris ${ }^{4}$ \\ ${ }^{1}$ Pulmonary/Critical Care Service, Department of Medicine, San Antonio Military Medical Center, JBSA Fort Sam Houston, TX 78234, USA \\ ${ }^{2}$ Pulmonary/Critical Care Service, Department of Medicine, Carl R. Darnall Army Medical Center, Fort Hood, TX, USA \\ ${ }^{3}$ Internal Medicine Residency, Department of Medicine, San Antonio Military Medical Center, JBSA Fort Sam Houston, TX, USA \\ ${ }^{4}$ Pulmonary/Critical Care Service, Department of Medicine, San Antonio Military Medical Center, JBSA Fort Sam Houston, TX, USA
}

\begin{abstract}
Background: The objective of this study was to investigate differences in Cardiopulmonary Exercise Testing (CPET) between deployed and non-deployed military personnel undergoing a clinical evaluation for dyspnea.

Methods: A retrospective electronic medical record review was conducted on active military personnel who underwent CPET at Brooke Army Medical Center during a clinical evaluation for dyspnea from 2007 through 2011. Studies were performed on a cycle ergometer to the point of maximum exercise tolerance. Review of CPET records identified values from expired gas analysis related to cardiac and respiratory exercise limitations and review of medical records identified pre-CPET diagnostic testing and medical and deployment history.

Results: A total of 268 patients were identified. The cohort was predominantly male (78\%) and had deployed to Southwest Asia (62\%). Demographic comparison of deployed and non-deployed groups showed age (32.2 vs. 30.5 years) and body mass index (28.1 vs. $26.6 \mathrm{~kg} \bullet \mathrm{m}-2)$ were higher in deployed personnel. Diffusing capacity for carbon monoxide was low in deployed and non-deployed personnel (71.1 vs. $75.0 \%$ predicted) and was significantly lower in deployed personnel with onset of dyspnea during or post-deployment ( $70.0 \%$ predicted). Anaerobic threshold was lower in deployed personnel (49.8 vs. $55.8 \%$ predicted) but there were no significant differences in maximum work rate, maximum oxygen consumption, maximum heart rate, heart rate response, maximum respiratory rate or other respiratory parameters.

Conclusions: Deployed males in this study were heavier and older than non-deployed males. There were small significant differences between the two groups in some CPET results (all within normal ranges); however, no specific CPET parameters were identified within this study that clearly defined an underlying pulmonary process related to deployment.
\end{abstract}

\section{Background}

Beginning with Operations Desert Shield/Desert Storm in the early 1990s and continuing through Operation Iraqi Freedom and Operation Enduring Freedom, military personnel returning from deployment to Southwest Asia (SWA) have reported new or worsening pulmonary symptoms. Ambient particulate matter levels from this region are elevated mainly due to sandstorms and geologic dusts, but other factors such as burn pit fumes, urban air pollution, vehicle exhaust and the increased rate of cigarette smoking may be environmental airborne hazards to deployed personnel [1]. The relationship between military deployment and these pulmonary symptoms has been the subject of numerous investigations $[2,3]$.

Several surveys have identified a higher incidence of pulmonary complaints in previously deployed personnel. The Iowa Persian Gulf Study Group noted a higher prevalence of self-reported asthma and bronchitis among surveyed veterans of the First Gulf War [4]. During the recent SWA conflicts, the Millennium Cohort Study found a higher rate of new self-reported respiratory symptoms when comparing deployed to non-deployed military members ( $14 \%$ vs. $10 \%)$, as well as a direct relationship between deployment length and the frequency of symptoms [5].

These deployment-related pulmonary symptoms have been investigated in the context of inhalational exposures. A 2002 study found the odds ratio for self-reported asthma and bronchitis was higher in veterans who reported oil fire smoke exposure [6]. Later studies described more self-reported pulmonary symptoms in veterans reporting exposures to oil fire smoke, dust storms and sulfur fires, but did not find diminished lung function or an increased rate of chronic respiratory conditions when compared to unexposed veterans $[7,8]$. Both the 2010 Armed Forces Health Surveillance Center report and 2011 Institute of Medicine report on the potential impacts of burn pits concluded there was no substantial or consistent health effects in personnel assigned near burn pit locations $[9,10]$.

Recent investigations have tried to connect inhalational exposure during military deployment to specific disease processes, including

Correspondence to: Paul D. Hiles, M.D, Capt. USAF, MC, Pulmonary/Critical Care Service, Department of Medicine, San Antonio Military Medical Center, JBSA Fort Sam Houston, TX 78234, USA, Tel: 703-470-5685; E-mail: paul.d.hiles. mil@mail.mil

Key words: exercise testing, military deployment, dyspnea and pulmonary function testing

Received: June 29, 2016; Accepted: August 13, 2016; Published: August 15, 2016 
asthma, acute eosinophilic pneumonia and constrictive bronchiolitis [2,3,11-14]. In patients with post-deployment respiratory symptoms, Cardiopulmonary Exercise Testing (CPET) has been utilized to potentially help identify small groups of individuals with normal or near normal pulmonary function testing for limitations to exercise $[2,15]$. No specific CPET parameters were identified that clearly defined an underlying pathophysiologic process related to deployment.

The objective of this study was to evaluate any identifiable relationship between military deployment to SWA and pulmonary symptoms by investigating the differences in CPET results between deployed and non-deployed personnel. Cardiopulmonary exercise testing can be an objective method of assessing functional capacity and may have a role in the evaluation of exertional dyspnea by revealing the underlying etiology, detecting early changes in patients with cardiac or respiratory disease, and determining the limiting disease process in an individual with multiple comorbidities $[16,17]$.

\section{Methods}

The Brooke Army Medical Center (BAMC) Institutional Review Board reviewed and approved this study. A retrospective review of BAMC Pulmonary Clinic records was conducted using the clinical CPET database and military electronic medical record. The requirement for informed consent was waived due to the retrospective nature of the study. Patients were included if they were Active Duty (AD) military personnel evaluated at the BAMC Pulmonary Clinic for complaints of dyspnea from 2007 through 2011 who completed a CPET using cycle ergometry. Retired military personnel and dependents were excluded, as were patients who completed a CPET study using a graded treadmill. Treadmill tests were excluded because cycle ergometers have been shown to more accurately quantify work rates and give less variable $\mathrm{VO}_{2}$ max values at submaximal work rates [18].

Patients were divided into two groups based on prior deployment history. Patients were defined as "deployed" if they deployed for at least three months to SWA from 2003 through 2011 before pulmonary and CPET evaluation, and as "non-deployed" if they had no deployment history or were deployed after pulmonary and CPET evaluation. All patients underwent an evaluation in the clinic to include pulmonary function testing and chest imaging. The remainder of the evaluation varied based on presenting symptoms and preference of the evaluating physician.

All patients had previously completed a standard CPET protocol consisting of exercise on an electromechanically braked cycle ergometer with an increasing workload of 20 watts per minute [18]. Heart rate (HR), blood pressure, 12-lead electrocardiogram, pulse oximetry, oxygen consumption $\left(\mathrm{VO}_{2}\right)$, carbon dioxide production $\left(\mathrm{VCO}_{2}\right)$ and expired ventilation $\left(\mathrm{V}_{\mathrm{E}}\right)$ were monitored during exercise and studies were terminated at the point of maximum exercise tolerance $[19,20]$. The following variables were included for analysis and compared with reference values listed in the American Thoracic Society/American College of Chest Physicians Statement on Cardiopulmonary Exercise Testing: exercise time, Maximum Heart Rate (max HR), Heart Rate Response (HRR), $\mathrm{VO}_{2}$ max, Anaerobic Threshold (AT), Maximum Respiratory Rate (RR max), tidal volume to inspiratory capacity ratio $\left(\mathrm{V}_{\mathrm{T}} / \mathrm{IC}\right)$, ventilatory equivalent for $\mathrm{CO}_{2}$ at anaerobic threshold $\left(\mathrm{V}_{\mathrm{E}} /\right.$ $\left.\mathrm{VCO}_{2}\right)$, ventilatory reserve $\left(\mathrm{V}_{\mathrm{E}} \max / \mathrm{MVV}\right)$ maximum work rate and oxygen pulse $\left(\mathrm{O}_{2}\right.$ pulse $)[21]$.

Patients' medical records were reviewed to determine the diagnostic pulmonary evaluation prior to CPET completion, including
Pulmonary Function Testing (PFT), Chest Radiography (CXR), Chest Computed Tomography (CT), Methacholine Challenge Testing (MCT), transthoracic echocardiogram, laryngoscopy, and bronchoscopy. Forced vital capacity (FVC), Forced Expiratory Volume at one second $\left(\mathrm{FEV}_{1}\right)$, total lung capacity (TLC), Residual Volume (RV), and Diffusion Capacity for Carbon Monoxide (DLCO) were recorded from available PFT data. Results of CXR, chest CT, MCT, echocardiogram, and bronchoscopy results were recorded as "normal" or "abnormal" based on documentation of any abnormalities in radiology or procedure reports. Laryngoscopy results were recorded as "normal" if Vocal Cord Dysfunction (VCD) was absent and "abnormal" if VCD was present based on documentation in the procedure note. The final diagnosis given for a patient's dyspnea was determined by reviewing Pulmonary Clinic notes. If the notes attributed symptoms to multiple diagnoses, all diagnoses were included.

The CPET and PFT results of the deployed and non-deployed groups were compared as the primary analysis. Results were then compared in three secondary analyses after stratifying the deployed group based on the 1) presence of a diagnosis for a patient's dyspnea 2) duration of deployment and 3) onset of dyspnea symptoms. Military deployment records were queried to separate deployed personnel into two groups: overall deployment length $<365$ days and overall deployment length $\geq 365$ days. Patients were not included in this analysis if deployment records were unavailable. The onset of dyspnea symptoms for deployed personnel was determined by reviewing Pulmonary Clinic notes. Patients were not included in this secondary analysis if there was no clear documentation of the onset of dyspnea. The percentage of abnormal CXR, chest CTs and other studies in the deployed and non-deployed groups were also compared.

Statistical analyses were performed with SAS version 9.3 software (SAS Institute Inc., Cary, NC) and p values were calculated using Chisquare, Wilcoxon Rank sum, T-test or Fischer Exact Test methods as appropriate for each reported variable. A $\mathrm{p}$ value less than 0.05 was considered significant.

\section{Results}

A total of 268 active duty military personnel fulfilled the inclusion criteria for analysis. From this group, 103 patients had not been deployed to SWA or were deployed after CPET evaluation ("nondeployed"), and 165 patients were deployed prior to CPET evaluation ("deployed"). There were more males in the deployed group (84 vs. 67, $\mathrm{p}<0.001)$, and patients in the deployed group were also older $(32.2 \pm$ 8.6 vs. $30.5 \pm 10.3$ years, $\mathrm{p}=0.02)$ with a higher body mass index $(28.1$ [IQR 25.1, 30.8] vs. 26.6 [IQR 24.1, 29.7] $\mathrm{kg} \cdot \mathrm{m}^{-2}, \mathrm{p}=0.02$ ). There were no significant differences in other demographics or smoking history, as shown in Table 1. Asthma was the most common diagnosis $(n=41)$, followed by sarcoidosis $(n=13)$, pectus excavatum $(n=11)$ and VCD $(\mathrm{n}=11)$ as shown in Table 2.

Among the patients with a chest CT performed, a higher percentage of non-deployed personnel had abnormal results ( $72 \mathrm{vs.} 54 \%, \mathrm{p}=0.024$ ), but there were no other significant differences in the percentage of diagnostic test abnormalities between the two groups (Table 3). In the non-deployed group, chest CT abnormalities included pectus excavatum ( 6 patients), a localized pulmonary infiltrate (5 patients), bronchial wall thickening (4 patients), hilar and/or mediastinal lymphadenopathy (4 patients), post-cardiac surgery changes (3 patients), apical fibrotic changes (2 patients), multiple pulmonary infiltrates ( 2 patients), cardiomegaly ( 2 patients), hyperinflation (2 patients), post-thoracic surgery changes (1 patient), a right-sided 
Table 1. Demographic Characteristics by Deployment History.

\begin{tabular}{|c|c|c|c|}
\hline Variable & $\begin{array}{l}\text { Non-Deployed } \\
(n=103)\end{array}$ & $\begin{array}{c}\text { Deployed } \\
(\mathrm{n}=165)\end{array}$ & Pvalue \\
\hline Gender, n (\%) & & & $<0.001^{1}$ \\
\hline Female & $34(33)$ & $26(16)$ & \\
\hline Male & $69(67)$ & $139(84)$ & \\
\hline Age & $30.5(10.3)$ & $32.2(8.6)$ & $0.02^{2}$ \\
\hline Height (in) & $69[66,71]$ & $69[67,71]$ & $0.17^{3}$ \\
\hline Weight (lbs) & $175[151,206]$ & $190[166,215]$ & $0.03^{3}$ \\
\hline BMI & $26.6[24.3,29.7]$ & $28.1[25.1,30.8]$ & $0.02^{3}$ \\
\hline Smoking, n (\%) & & & $0.23^{1}$ \\
\hline No & $69(67)$ & $101(61)$ & \\
\hline Former & $15(15)$ & $38(23)$ & \\
\hline Active & $19(18)$ & $26(16)$ & \\
\hline Total & 103 & 165 & \\
\hline
\end{tabular}

${ }^{1}$ Chi-square; ${ }^{2}$ Wilcoxon Rank sum; ${ }^{3}$ T-test

Continuous Variables are reported as Mean (SD) or Median [IQR] based on normality of distribution

$\mathrm{BMI}=\left(\mathrm{lbs} / \mathrm{ht}^{2}\right) * 703$

Table 2. Diagnoses of Dyspnea by Deployment History.

\begin{tabular}{|c|c|c|}
\hline Diagnosis & Non-Deployed & Deployed \\
\hline Asthma & 14 & 27 \\
\hline Sarcoidosis & 6 & 7 \\
\hline Pectus excavatum & 11 & 0 \\
\hline Vocal cord dysfunction & 3 & 8 \\
\hline Obstructive lung disease $^{1}$ & 5 & 8 \\
\hline Diffuse parenchymal lung disease ${ }^{2}$ & 2 & 8 \\
\hline Pulmonary vascular disease ${ }^{3}$ & 1 & 4 \\
\hline Pleural disease ${ }^{4}$ & 0 & 2 \\
\hline Cardiovascular disease $^{5}$ & 3 & 0 \\
\hline Occupational $^{6}$ & 1 & 6 \\
\hline Acute eosinophilic pneumonia & 1 & 0 \\
\hline Bronchogenic cyst & 1 & 0 \\
\hline Deconditioning & 1 & 3 \\
\hline Diaphragm weakness & 1 & 2 \\
\hline Obstructive sleep apnea & 0 & 1 \\
\hline Pulmonary nodules & 1 & 0 \\
\hline Tuberculosis & 1 & 0 \\
\hline
\end{tabular}

${ }^{1}$ Includes allergic bronchopulmonary aspergillosis, bronchiectasis, bronchiolitis, COPD, exercise-induced bronchospasm, reactive airway dysfunction syndrome

${ }^{2}$ Includes Langerhans cell histiocytosis, systemic lupus erythematosus, hypersensitivity pneumonitis, drug-induced parenchymal lung disease, radiation-induced parenchymal lung disease, lymphangioleiomyomatosis, and idiopathic interstitial lung disease

3 Includes pulmonary embolism, pulmonary hypertension, pulmonary arteriovenous malformation

${ }^{4}$ Includes pleural effusion, pneumothorax

${ }^{5}$ Includes heart failure, aortic valve disease, mitral valve prolapse

${ }^{6}$ Includes inhalational exposure (dust, burn pits, smoke, "toxic cloud"), burns, trauma aorta (1 patient), pneumothorax (1 patient), pulmonary nodules (1 patient), a prominent left heart border (1 patient), interstitial lung disease (1 patient), prior granulomatous disease (1 patient), an elevated left hemidiaphragm (1 patient), scoliosis (1 patient) and findings suggestive of sarcoidosis (1 patient). In the deployed group, chest CT abnormalities included post-thoracic surgery changes (10 patients), hyperinflation (6 patients), a localized pulmonary infiltrate (5 patients), hilar and/or mediastinal lymphadenopathy (4 patients), interstitial lung disease ( 3 patients), pulmonary nodules ( 3 patients), prior granulomatous disease ( 3 patients), scarring ( 2 patients), pectus excavatum ( 2 patients), pneumothorax (2 patients), pleural thickening (2 patients), multiple pulmonary infiltrates (1 patient), bronchial wall thickening (1 patient), atelectasis (1 patient), diaphragm tenting (1 patient), and a pleural effusion (1 patient).

Table 4 displays the CPET and PFT results for the deployed and non-deployed groups, as well as the results after stratification by duration of deployment. Deployed personnel had a significantly lower $\mathrm{FEV}_{1}(86.0 \pm 15.2$ vs. $91.5 \pm 14.2 \%$ predicted, $\mathrm{p}=0.005)$ and FVC $(83.2 \pm 15.8$ vs. $87.4 \pm 14.8 \%$ predicted, $\mathrm{p}=0.04)$ compared to non-deployed personnel. Both groups had an abnormally low DLCO: $71.1 \%$ predicted for deployed personnel and $75.0 \%$ predicted for nondeployed personnel. Personnel deployed $\geq 365$ days had a lower FEV than personnel deployed $<365$ days and non-deployed personnel (85.7 \pm 12.5 vs. $86.4 \pm 17.6$ vs. $91.5 \pm 14.2 \%$ predicted, $\mathrm{p}=0.02$ ), but there were no significant differences in any other PFT measurements.

Both the deployed and non-deployed groups had similar max $\mathrm{HR}$ $(89.1 \pm 8.7$ and $89.5 \pm 7.6 \%$ predicted, $\mathrm{p}=0.72)$ and a reduced $\mathrm{VO}_{2} \max$ (78.8 \pm 18.4 and $79.4 \pm 18.1 \%$ predicted, $\mathrm{p}=0.78)$. The only statistically significant difference was a lower AT in the deployed group (49.8 \pm 17.3 vs. $55.8 \pm 18.0 \%$ predicted, $\mathrm{p}=0.007)$. Non-deployed personnel had a significantly higher HRR compared to personnel deployed $<365$ days and personnel deployed $\geq 365$ days $(47.7 \pm 14.9$ vs. $47.3 \pm 14.6$ vs. 42.1 $\pm 13.7, \mathrm{p}=0.01)$. There was also a significantly lower AT in personnel deployed $<365$ days compared to personnel deployed $\geq 365$ days and non-deployed personnel $(47.9 \pm 14.9$ vs. $51.5 \pm 19.1$ vs. $55.8 \pm 18.0 \%$ predicted, $\mathrm{p}=0.01$ ).

When stratified by the presence of a diagnosis causing dyspnea, deployed personnel with a diagnosis had a significantly lower AT compared to deployed personnel without a diagnosis and nondeployed personnel $(48.2 \pm 15.4$ vs. $51.1 \pm 18.6$ vs. $55.8 \pm 18.0 \%$ predicted, $\mathrm{p}=0.02$ ) (Table 5). There was a significantly higher $\mathrm{V}_{\mathrm{E}} / \mathrm{VCO}_{2}$ in deployed personnel with a diagnosis compared to non-deployed personnel and deployed personnel without a diagnosis (29 [IQR 26, 31] vs. 27 [IQR 25, 30] vs. 26 [IQR 25, 29], p=0.008). Deployed personnel with a diagnosis had lower $\mathrm{FEV}_{1}, \mathrm{FVC}$, and $\mathrm{FEV}_{1} / \mathrm{FVC}$ values that were statistically significant when compared to non-deployed personnel and deployed personnel without a diagnosis.

Table 3. Diagnostic Test Abnormalities by Deployment History.

\begin{tabular}{|c|c|c|c|c|c|c|c|}
\hline \multirow[b]{2}{*}{ Diagnostic Test } & \multicolumn{3}{|c|}{ Non-Deployed } & \multicolumn{3}{|c|}{ Deployed } & \multirow[b]{2}{*}{ Pvalue $^{1}$} \\
\hline & Abnormal (n) & Total (n) & Percent Abnormal (\%) & Abnormal (n) & Total (n) & $\begin{array}{c}\text { Percent } \\
\text { Abnormal (\%) }\end{array}$ & \\
\hline Chest x-ray & 37 & 94 & 39 & 44 & 158 & 28 & 0.070 \\
\hline Chest CT & 44 & 61 & 72 & 64 & 119 & 54 & 0.024 \\
\hline Methacholine & 11 & 47 & 23 & 12 & 75 & 16 & 0.35 \\
\hline Echocardiogram & 32 & 52 & 62 & 38 & 84 & 45 & 0.078 \\
\hline Laryngoscopy & 4 & 22 & 18 & 8 & 32 & 25 & 0.74 \\
\hline Bronchoscopy & 5 & 7 & 71 & 6 & 8 & 75 & 1.0 \\
\hline
\end{tabular}

${ }^{1}$ Fischer Exact Test 
Table 4. CPET and PFT Results by Deployment History and Duration of Deployment.

\begin{tabular}{|c|c|c|c|c|c|c|c|}
\hline Variable & $\begin{array}{l}\text { Reference } \\
\text { Value }\end{array}$ & $\begin{array}{l}\text { Non-Deployed } \\
\quad(n=103)\end{array}$ & $\begin{array}{c}\text { Deployed } \\
(n=165)\end{array}$ & P-value & $\begin{array}{c}\text { Deployed }<365 \text { days } \\
(n=77)\end{array}$ & $\begin{array}{c}\text { Deployed } \geq 365 \text { days } \\
(n=88)\end{array}$ & P value \\
\hline Exercise time (min) & None & $9.67(2.47)$ & $9.60(2.11)$ & $0.82^{1}$ & $9.36(2.04)$ & $9.81(2.16)$ & $0.42^{1}$ \\
\hline Max HR (\% predicted) & $>90$ & $89.5(7.6)$ & $89.1(8.7)$ & $0.72^{1}$ & $89.0(8.1)$ & $89.2(9.2)$ & $0.92^{1}$ \\
\hline Heart rate response & $\leq 50$ & $47.7(14.9)$ & $44.5(14.3)$ & $0.08^{1}$ & $47.3(14.6)$ & $42.1(13.7)$ & $0.01^{1}$ \\
\hline $\mathrm{VO}_{2} \max (\%$ predicted $)$ & $>84$ & $79.4(18.1)$ & $78.8(18.4)$ & $0.78^{1}$ & $76.4(17.3)$ & $80.9(19.2)$ & $0.27^{1}$ \\
\hline $\mathrm{AT}\left(\%\right.$ predicted $\left.\mathrm{VO}_{2} \max \right)$ & $>40$ & $55.8(18.0)$ & $49.8(17.3)$ & $0.007^{1}$ & $47.9(14.9)$ & $51.5(19.1)$ & $0.01^{1}$ \\
\hline RR max (breaths/min) & $<60$ & $39[34,45]$ & $37[31,46]$ & $0.47^{2}$ & $37[31,48]$ & $37[32,44]$ & $0.73^{2}$ \\
\hline $\mathrm{V}_{\mathrm{T}} / \mathrm{IC}$ & $<0.80$ & $0.63[0.54,0.74]$ & $0.68[0.57,0.80]$ & $0.1^{2}$ & $0.65[0.52,0.75]$ & $0.71[0.58,0.82]$ & $0.05^{2}$ \\
\hline $\mathrm{V}_{\mathrm{E}} / \mathrm{VCO}_{2}$ (at AT) & $<34$ & $27[25,30]$ & $27[25,30]$ & $0.62^{2}$ & $27[25,29]$ & $27[25,30]$ & $0.82^{2}$ \\
\hline $\mathrm{V}_{\mathrm{E}} \max / \mathrm{MVV}$ & $0.72 \pm 0.12$ & $0.62[0.53,0.77]$ & $0.65[0.51,0.76]$ & $0.97^{2}$ & $0.62[0.49,0.76]$ & $0.68[0.54,0.78]$ & $0.42^{2}$ \\
\hline Max work rate (watts) & None & $183.85(53.72)$ & $184.74(42.94)$ & $0.88^{1}$ & $178.49(40.62)$ & $190.28(44.39)$ & $0.28^{1}$ \\
\hline $\mathrm{O}_{2}$ pulse (\% predicted) & $>80$ & $86.8[75.8,99.6]$ & $88.0[73.1,101.8]$ & $0.98^{2}$ & $85.6[73.0,95.6]$ & $90.8[74.0,108.4]$ & $0.29^{2}$ \\
\hline $\mathrm{FEV}_{1}(\%$ predicted $)$ & $80-120$ & $91.5(14.2)$ & $86.0(15.2)$ & $0.005^{1}$ & 86.4 (17.6) & $85.7(12.5)$ & $0.02^{1}$ \\
\hline FVC (\% predicted $)$ & $80-120$ & $87.4(14.8)$ & $83.2(15.8)$ & $0.04^{1}$ & $82.9(17.8)$ & $83.4(13.7)$ & $0.11^{1}$ \\
\hline $\mathrm{FEV}_{1} / \mathrm{FVC}$ (actual) & $>70$ & $80.2[77.2,82.9]$ & $80.1[75.5,84.2]$ & $0.78^{2}$ & $79.3[75.7,84.0]$ & $81.3[75.3,84.3]$ & $0.71^{2}$ \\
\hline TLC (\% predicted) & $80-120$ & $89.4(15.6)$ & $86.9(15.8)$ & $0.33^{1}$ & $84.5(15.1)$ & $88.8(16.2)$ & $0.24^{1}$ \\
\hline DLCO $(\%$ predicted $)$ & $80-120$ & $75.0[66.5,86.3]$ & $71.1[63.5,81.8]$ & $0.12^{2}$ & $70.0[63.1,82.8]$ & $71.5[63.6,79.7]$ & $0.3^{2}$ \\
\hline
\end{tabular}

${ }^{1}$ T-test

${ }^{2}$ Wilcoxon Rank sum

Continuous Variables are reported as Mean (SD) or Median [IQR] based on normality of distribution

Table 5: CPET and PFT Results by Presence of a Diagnosis.

\begin{tabular}{|c|c|c|c|c|c|}
\hline Variable & Reference Value & $\begin{array}{l}\text { Non-Deployed } \\
\quad(n=103)\end{array}$ & $\begin{array}{l}\text { Deployed with Diagnosis } \\
(n=73)\end{array}$ & $\begin{array}{l}\text { Deployed without Diagnosis } \\
(\mathrm{n}=92)\end{array}$ & Pvalue \\
\hline Exercise time (min) & None & $9.67(2.47)$ & $9.16(2.06)$ & $9.95(2.10)$ & $0.08^{1}$ \\
\hline Max HR (\% predicted) & $>90$ & $89.5(7.6)$ & $89.1(8.7)$ & $89.1(9.6)$ & $0.94^{1}$ \\
\hline Heart rate response & $\leq 50$ & $47.7(14.9)$ & $45.6(14.0)$ & $43.7(14.6)$ & $0.15^{1}$ \\
\hline $\mathrm{VO}_{2} \max (\%$ predicted $)$ & $>84$ & $79.4(18.1)$ & $76.5(17.9)$ & $80.6(18.7)$ & $0.34^{1}$ \\
\hline AT $\left(\%\right.$ predicted $\left.\mathrm{VO}_{2} \max \right)$ & $>40$ & $55.8(18.0)$ & $48.2(15.4)$ & $51.1(18.6)$ & $0.02^{1}$ \\
\hline RR max (breaths/min) & $<60$ & $39[34,45]$ & $40[30,51]$ & $36[32,42]$ & $0.19^{2}$ \\
\hline $\mathrm{V}_{\mathrm{T}} / \mathrm{IC}$ & $<0.80$ & $0.63[0.54,0.74]$ & $0.67[0.50,0.76]$ & $0.69[0.59,0.82]$ & $0.07^{2}$ \\
\hline $\mathrm{V}_{\mathrm{E}} / \mathrm{VCO}_{2}$ (at $\mathrm{AT}$ ) & $<34$ & $27[25,30]$ & $29[26,31]$ & $26[25,29]$ & $0.008^{2}$ \\
\hline $\mathrm{V}_{\mathrm{E}} \max / \mathrm{MVV}$ & $0.72 \pm 0.12$ & $0.62[0.53,0.77]$ & $0.66[0.52,0.81]$ & $0.65[0.51,0.73]$ & $0.77^{2}$ \\
\hline Max work rate (watts) & None & $183.85(53.72)$ & $177.79(40.86)$ & $190.32(43.96)$ & $0.24^{1}$ \\
\hline $\mathrm{O}_{2}$ pulse (\% predicted) & $>80$ & $86.8[75.8,99.6]$ & $84.3[70.6,101.3]$ & $89.2[79.4,103.0]$ & $0.29^{2}$ \\
\hline $\mathrm{FEV}_{1}(\%$ predicted $)$ & $80-120$ & $91.5(14.2)$ & $84.4(13.1)$ & $87.4(16.6)$ & $0.009^{1}$ \\
\hline FVC $(\%$ predicted $)$ & $80-120$ & $87.4(14.8)$ & $79.9(15.1)$ & $85.7(16.0)$ & $0.007^{1}$ \\
\hline $\mathrm{FEV}_{1} / \mathrm{FVC}$ (actual) & $>70$ & $80.2[77.2,82.9]$ & $78.4[73.8,83.6]$ & $82.1[77.6,84.5]$ & $0.03^{2}$ \\
\hline TLC (\% predicted) & $80-120$ & $89.4(15.6)$ & $87.0(13.2)$ & $86.8(17.9)$ & $0.62^{1}$ \\
\hline DLCO $(\%$ predicted $)$ & $80-120$ & $75.0[66.5,86.3]$ & $70.0[63.4,80.8]$ & $71.6[63.6,82.0]$ & $0.28^{2}$ \\
\hline
\end{tabular}

${ }^{1}$ T-test; ${ }^{2}$ Wilcoxon Rank sum

Continuous Variables are reported as Mean (SD) or Median [IQR] based on normality of distribution

Stratification of CPET results by the onset of dyspnea revealed that non-deployed personnel had a significantly higher HRR compared to deployed personnel with onset pre-deployment and deployed personnel with onset during or post-deployment $(47.7 \pm 14.9$ vs. $47.1 \pm 14.3$ vs. $42.4 \pm 12.9, \mathrm{p}=0.04$ ) (Table 6). Anaerobic threshold was significantly lower in deployed personnel with onset pre-deployment compared to deployed personnel with onset during or post-deployment and non-deployed personnel $(45.3 \pm 20.8$ vs. $52.1 \pm 16.4$ vs. $55.8 \pm 18.0 \%$ predicted, $\mathrm{p}=0.02$ ). Deployed personnel with onset of dyspnea during or post-deployment had significantly lower DLCO values than nondeployed personnel or personnel with onset pre-deployment $(70.0$ [IQR 63.6, 77.6] vs. 75.0 [IQR 66.5, 86.3] vs. 75.7 [IQR 68.4, 93.5] \% predicted, $\mathrm{p}=0.03$ ). The medical record did not specify the onset of dyspnea for 57 deployed personnel, so those patients were excluded from this secondary analysis.

\section{Discussion}

The intent of this study was to evaluate the relationship between military deployment and pulmonary symptoms by comparing the CPET results of deployed and non-deployed personnel undergoing an evaluation for dyspnea. The primary analysis revealed no significant differences in CPET results between the two groups, with the exception of a lower AT in the deployed group that was within the accepted range of normal based on established reference values [21]. The $\mathrm{VO}_{2}$ max was lower than predicted in both groups; however, this is not unexpected in a group of patients being evaluated for dyspnea.

Demographic differences may have affected the ability to detect a significant difference in $\mathrm{VO}_{2}$ max and max $\mathrm{HR}$, as deployed personnel were older and $\mathrm{VO}_{2}$ max and max HR linearly regress with age [22-24]. The increased weight and BMI in the deployed group could have had a similar effect, as peak $\mathrm{VO}_{2}$ max prediction is also dependent on weight 
Table 6: CPET and PFT Results by Onset of Dyspnea.

\begin{tabular}{|c|c|c|c|c|c|}
\hline Variable & Reference Value & $\begin{array}{l}\text { Non-Deployed } \\
(n=103)\end{array}$ & $\begin{array}{l}\text { Deployed with Onset } \\
\text { Pre-deployment } \\
(n=34)\end{array}$ & $\begin{array}{l}\text { Deployed with Onset During/ } \\
\text { Post-deployment } \\
(\mathrm{n}=74)\end{array}$ & P value \\
\hline Exercise time (min) & None & $9.67(2.47)$ & $9.15(2.31)$ & $9.81(1.97)$ & $0.41^{1}$ \\
\hline Max HR (\% predicted) & $>90$ & $89.5(7.6)$ & $85.7(9.1)$ & $89.9(8.0)$ & $0.05^{1}$ \\
\hline Heart rate response & $\leq 50$ & $47.7(14.9)$ & $47.1(14.3)$ & $42.4(12.9)$ & $0.04^{1}$ \\
\hline $\mathrm{VO}_{2} \max (\%$ predicted $)$ & $>84$ & $79.4(18.1)$ & $77.7(22.9)$ & $81.2(17.4)$ & $0.65^{1}$ \\
\hline AT $\left(\%\right.$ predicted $\left.\mathrm{VO}_{2} \max \right)$ & $>40$ & $55.8(18.0)$ & $45.3(20.8)$ & $52.1(16.4)$ & $0.02^{1}$ \\
\hline RR max (breaths/min) & $<60$ & $39[34,45]$ & $37[30,55]$ & $38[35,44]$ & $0.92^{2}$ \\
\hline $\mathrm{V}_{\mathrm{T}} / \mathrm{IC}$ & $<0.80$ & $0.63[0.54,0.74]$ & $0.69[0.54,0.84]$ & $0.66[0.57,0.76]$ & $0.58^{2}$ \\
\hline $\mathrm{V}_{\mathrm{E}} / \mathrm{VCO}_{2}$ (at AT) & $<34$ & $27[25,30]$ & $28[26,32]$ & $27[25,30]$ & $0.17^{2}$ \\
\hline $\mathrm{V}_{\mathrm{E}} \max / \mathrm{MVV}$ & $0.72 \pm 0.12$ & $0.62[0.53,0.77]$ & $0.65[0.46,0.78]$ & $0.67[0.55,0.75]$ & $0.46^{2}$ \\
\hline Max work rate (watts) & None & $183.85(53.72)$ & $174.63(47.74)$ & $191.78(41.08)$ & $0.25^{1}$ \\
\hline $\mathrm{O}_{2}$ pulse (\% predicted) & $>80$ & $86.8[75.8,99.6]$ & $87.3[74.7,107.7]$ & $90.4[79.6,102.4]$ & $0.76^{2}$ \\
\hline $\mathrm{FEV}_{1}(\%$ predicted $)$ & $80-120$ & $91.5(14.2)$ & $89.0(17.5)$ & $86.6(13.8)$ & $0.1^{1}$ \\
\hline FVC $(\%$ predicted $)$ & $80-120$ & $87.4(14.8)$ & $86.7(17.0)$ & $84.3(14.6)$ & $0.41^{1}$ \\
\hline $\mathrm{FEV}_{\mathrm{l}} / \mathrm{FVC}$ (actual) & $>70$ & $80.2[77.2,82.9]$ & $80.5[77.8,84.6]$ & $81.3[74.6,84.8]$ & $0.72^{2}$ \\
\hline TLC $(\%$ predicted $)$ & $80-120$ & $89.4(15.6)$ & $85.3(9.7)$ & $88.2(17.6)$ & $0.7^{1}$ \\
\hline DLCO $(\%$ predicted $)$ & $80-120$ & $75.0[66.5,86.3]$ & $75.7[68.4,93.5]$ & $70.0[63.6,77.6]$ & $0.03^{2}$ \\
\hline
\end{tabular}

${ }^{1}$ T-test; ${ }^{2}$ Wilcoxon Rank sum

Continuous Variables are reported as Mean (SD) or Median [IQR] based on normality of distribution

[19,25-27]. If these demographic differences did have an impact, they could have lowered both the $\mathrm{VO}_{2}$ max and max $\mathrm{HR}$ in the deployed group. The age difference would not have been expected to significantly impact cardiovascular and respiratory variables, as the change in those variables with age is minimal until age $50[27,28]$. Additionally, the number of cigarette smokers in the two groups was not significantly different, which is important because smoking may impact CPET respiratory variables [23].

The three secondary analyses revealed some statistically significant differences in $\mathrm{AT}, \mathrm{V}_{\mathrm{E}} / \mathrm{VCO}_{2}, \mathrm{HRR}$ and max $\mathrm{HR}$, but the values for these variables were all within the accepted range of normal [20]. Although these differences were statistically significant, they are not likely clinically significant because the mean values were still within predicted range of normal. While deployment length has been associated with increased pulmonary symptoms, this study did not identify an impact of deployment length on CPET results [5]. The similar CPET results among deployed personnel stratified by the onset of dyspnea argue against a significant sustained impact from potential inhalational exposures experienced during deployment $[2,6,8]$. Deployed personnel were specifically stratified by the presence of a dyspnea diagnosis to look for a pattern of CPET or PFT abnormalities among deployed personnel without a diagnosis, but the results for this group revealed a decreased $\mathrm{VO}_{2}$ max, minimally decreased max $\mathrm{HR}$, and low DLCO, not suggestive of any particular disease process.

Pulmonary function data were notable for lower, but still normal $\mathrm{FEV}_{1}$ and FVC values in the deployed group. Deployed personnel with a specific diagnosis had a significantly lower and slightly decreased FVC; this is not an unexpected finding in patients with established pulmonary disease. The DLCO was significantly lower in deployed personnel with onset of dyspnea during or post-deployment, which could indicate the development of interstitial or emphysematous lung changes related to inhalational exposures during deployment; however, this finding did not correlate with a reduction in TLC or the presence of imaging abnormalities, and there was actually a significantly higher percentage of abnormal chest CTs in non-deployed personnel. Additionally, other PFT values in the secondary analysis were not suggestive of an obstructive or restrictive process. There were 13 more diagnoses of asthma in deployed personnel, but asthma is typically associated with a normal or increased DLCO [29].

Cardiopulmonary exercise testing may not be the ideal modality to diagnose the etiology of a patient's dyspnea in this population. The 2002 study by Morris et al. of 105 active duty patients with exertional dyspnea concluded that CPET added little to the diagnostic evaluation compared to pulmonary function tests, methacholine challenge, laryngoscopy and chest imaging [15]. A 2009 study of the same cohort used age-matched controls when interpreting CPET results of the patients with exertional dyspnea. Compared to established reference values using age-matched controls resulted in statistically significant differences in the sensitivity ( $53 \%$ vs. $12 \%)$ and specificity ( $31 \%$ vs. 96\%) of $\mathrm{V}_{\mathrm{T}} / \mathrm{IC}$, which suggests CPET may be insensitive in detecting mild pulmonary disease in young healthy adults [30]. Finally, the 2011 King study of deployed military personnel found to have constrictive bronchiolitis on surgical lung biopsy utilized CPET in some of the patients evaluated and there were significant decreases in $\mathrm{VO}_{2}$ max and AT (compared to published normal controls) but no increase in measured respiratory parameters [2].

This study has several limitations. The retrospective design subjects the study to confounding variables. There is a lack of final diagnoses in many patients and incomplete data sets to ideally compare differences between groups. Due to the lack of documentation of specific inhalational exposures, onset of dyspnea symptoms was used as a surrogate measure to investigate the effect of any deployment exposures, which is not an exact substitute. Lastly, the diagnostic utility of CPET is not absolute, as previous studies have demonstrated that it has a limited diagnostic yield, especially in young healthy adults with mild disease [30]. Both the 2002 Morris et al. study of exertional dyspnea and the recent 2014 STAMPEDE study are examples of this, as a diagnosis was not established in $25 \%$ and $42 \%$ of patients in those studies, respectively $[3,15]$.

\section{Conclusions}

This review of CPET values in military personnel evaluated for dyspnea revealed small significant differences in specific CPET 
values between deployed and non-deployed personnel; however, the values in both groups were within normal limits (based on published reference values) and CPET interpretation did not indicate an increase in pulmonary abnormalities in the deployed population. The utility of CPET evaluation in this population may be limited and may only establish exercise capacity. Future prospective trials investigating CPET in the relationship between military deployment and pulmonary symptoms are warranted to help further characterize any association.

\section{Authors' contributions}

Dr. Hiles conducted the electronic medical record review and drafted the manuscript. Dr. Morris designed the study and reviewed and edited the manuscript. Dr. Porr and Dr. Hannah reviewed and edited the manuscript.

\section{Acknowledgements}

The authors would like to thank LeeAnn Zarzabal for performing the statistical analysis.

\section{Competing interest}

Dr. Morris is part of the Speaker's Bureau for Spiriva (BoehringerIngelheim). Dr. Hiles, Dr. Porr and Dr. Hannah have no competing interests.

\section{Disclosure}

The view(s) expressed herein are those of the author(s) and do not reflect the official policy or position of Brooke Army Medical Center, the U.S. Army Medical Department, the U.S. Army Office of the Surgeon General, the Department of the Army, the Department of the Air Force and Department of Defense or the U.S. Government.

\section{References}

1. Weese CB, Abraham JH (2009) Potential health implications associated with particulate matter exposure in deployed settings in southwest Asia. Inhal Toxicol 21: 291-296.

2. King MS, Eisenberg R, Newman JH, Tolle JJ, Harrell FE Jr, et al. (2011) Constrictive bronchiolitis in soldiers returning from Iraq and Afghanistan. N Engl J Med 365: 222 230. [Crossref]

3. Morris MJ, Dodson DW, Lucero PF, Haislip GD, Gallup RA, et al. (2014) Study of active duty military for pulmonary disease related to environmental deployment exposures (STAMPEDE). Am J Respir Crit Care Med 190: 77-84. [Crossref]

4. [No authors listed] (1997) Self-reported illness and health status among Gulf War veterans. A population-based study. The Iowa Persian Gulf Study Group. JAMA 277: 238-245. [Crossref]

5. Smith B, Wong CA, Smith TC, Boyko EJ, Gackstetter GD, et al. (2009) Newly reported respiratory symptoms and conditions among military personnel deployed to Iraq and Afghanistan: a prospective population-based study. Am J Epidemiol 170: 1433-1442.

6. Lange JL, Schwartz DA, Doebbeling BN, Heller JM, Thorne PS (2002) Exposures to the Kuwait oil fires and their association with asthma and bronchitis among gulf war veterans. Environ Health Perspect 110: 1141-1146. [Crossref]

7. Baird CP, DeBakey S, Reid L, Hauschild VD, Petruccelli B, et al. (2012) Respiratory health status of US Army personnel potentially exposed to smoke from $2003 \mathrm{Al}$ Mishraq sulfur plant fire. J Occup Environ Med 54: 717-23. [Crossref]

8. Kelsall HL, Sim MR, Forbes AB, McKenzie DP, Glass DC, et al. (2004) Respiratory health status of Australian veterans of the 1991 Gulf War and the effects of exposure to oil fire smoke and dust storms. Thorax 59: 897-903. [Crossref]

9. Armed Forces Health Surveillance Center. Epidemiological Studies of Health Outcomes among Troops Deployed to Burn Pit Sites. Silver Spring, MD: Department of Defense; 2010.58 p.

10. Institute of Medicine. Long-term health consequences of exposure to burn pits in Iraq and Afghanistan. Washington, DC: The National Academies Press; 2011. 180 p.

11. Roop SA, Niven AS, Calvin BE, Bader J, Zacher LL (2007) The prevalence and impact of respiratory symptoms in asthmatics and nonasthmatics during deployment. Mil Med 172: 1264-1269. [Crossref]

12. Szema AM, Peters MC, Weissinger KM, Gagliano CA, Chen JJ (2010) New-onset asthma among soldiers serving in Iraq and Afghanistan. Allergy Asthma Proc 31: 6771. [Crossref]

13. Centers for Disease Control and Prevention (2003) Severe acute pneumonitis among deployed U.S. military personnel--Southwest Asia, March-August 2003. MMWR Morb Mortal Wkly Rep 52: 857-859. [Crossref]

14. Shorr AF, Scoville SL, Cersovsky SB, Shanks GD, Ockenhouse CF, et al. (2004) Acute eosinophilic pneumonia among US Military personnel deployed in or near Iraq. JAMA 292: 2997-3005. [Crossref]

15. Morris MJ, Grbach VX, Deal LE, Boyd SY, Morgan JA, et al. (2002) Evaluation of exertional dyspnea in the active duty patient: the diagnostic approach and the utility of clinical testing. Mil Med 167: 281-288. [Crossref]

16. McKelvie RS, Jones NL (1989) Cardiopulmonary exercise testing. Clin Chest Med 10 277-291. [Crossref]

17. Wasserman K. Principles of exercise testing and interpretation : including pathophysiology and clinical applications. 4th ed. Philadelphia: Lippincott Williams \& Wilkins; 2005: 585. [Crossref]

18. Storer TW, Davis JA, Caiozzo VJ (1990) Accurate prediction of VO2max in cycle ergometry. Med Sci Sports Exerc 22: 704-712. [Crossref]

19. Hansen JE, Sue DY, Wasserman K (1984) Predicted values for clinical exercise testing. Am Rev Respir Dis 129: S49-55. [Crossref]

20. Jones NL, Makrides L, Hitchcock C, Chypchar T, McCartney N (1985) Norma standards for an incremental progressive cycle ergometer test. Am Rev Respir Dis 131: 700-708. [Crossref]

21. American Thoracic Society; American College of Chest Physicians (2003) ATS/ACCP Statement on cardiopulmonary exercise testing. Am J Respir Crit Care Med 167: 211277. [Crossref]

22. Froelicher VF Jr, Allen M, Lancaster MC (1974) Maximal treadmill testing of normal USAF aircrewmen. Aerosp Med 45: 310-315. [Crossref]

23. Inbar O, Oren A, Scheinowitz M, Rotstein A, Dlin R, et al. (1994) Normal cardiopulmonary responses during incremental exercise in 20- to 70-yr-old men. Med Sci Sports Exerc 26: 538-46. [Crossref]

24. Vogel JA, Patton JF, Mello RP, Daniels WL (1986) An analysis of aerobic capacity in a large United States population. J Appl Physiol 60: 494-500. [Crossref]

25. Bruce RA, Kusumi F, Hosmer D 1973 Maximal oxygen intake and nomographic assessment of functional aerobic impairment in cardiovascular disease. Am Heart $J$ 85: 546-62. [Crossref]

26. Fairbarn MS, Blackie SP, McElvaney NG, Wiggs BR, Paré PD, et al. (1994) Prediction of heart rate and oxygen uptake during incremental and maximal exercise in healthy adults. Chest 105: 1365-1369. [Crossref]

27. Neder JA, Nery LE, Castelo A, Andreoni S, Lerario MC, et al. (1999) Prediction of metabolic and cardiopulmonary responses to maximum cycle ergometry: a randomised study. Eur Respir J 14: 1304-1313. [Crossref]

28. Drinkwater BL, Horvath SM, Wells CL (1975) Aerobic power of females, ages 10 to 68. J Gerontol 30: 385-394. [Crossref]

29. Saydain G, Beck KC, Decker PA, Cowl CT, Scanlon PD (2004) Clinical significance of elevated diffusing capacity. Chest 125: 446-452. [Crossref]

30. Sill JM, Morris MJ, Johnson JE, Allan PF, Grbach VX (2009) Cardiopulmonary exercise test interpretation using age-matched controls to evaluate exertional dyspnea. Mil Med 174: 11177-82. [Crossref]

Copyright: $(2016$ Hiles PD. This is an open-access article distributed under the terms of the Creative Commons Attribution License, which permits unrestricted use, distribution, and reproduction in any medium, provided the original author and source are credited. 